\title{
Studien über das Gel der Kieselsäure.
}

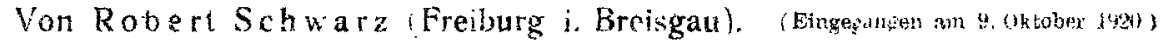

In den letaten Jahren habe ich eine Reihe von Untersuchungen vorgenommen, die sich mit dem AuflösungsprozeB der Kieselsäure in wässerigem Ammoniak beschäftigen. Die Resultate, aus denen sich unter anderem die Existenz von Ammoniumsalzen der Kieselsăure ergibt, sind in einer Anzahl von Abhandiungen ) wiedergegeben worden. Bei einer Materie wie der Kieselsäure zeigten sich nun, wie nicht anders zu erwarten, bej fortschreitender Beschäftigung auf diesem Gebiete mebr und mehr solche Probleme, die weniger rein chemischen als vielmehr kolloidchemischen. Charakter iositzen. Aus diesem Grunde scheint es gegeben, an dieser Stelle eine zusammenfassende Diskussion der bisherigen Ergebnisse der Untersuchungen vorzunehmen. Die teilweise recht umfangreichen Tabellen mit den Versuchsdaten sind im Interesse der Raumersparnis hier nicht noch einmal wiedergegeben. Ihretwegen sei auf die genannien Veröffentlichungen hingewiesen.

$\mathrm{DaB}$ sich wasserhaltige Kieselsäure in Ammoniakwasser zu lösen vermag, ist bereits frühzeitig festgestellt ${ }^{2}$ ) und von mehreren Autoren weiterhin bestätigt worden ${ }^{3}$. Bei allen Bestimnungen dieser Art handeite es sich freiiich stets nur um die Feststellung derienigen "löslichen" Kieselsäure, die ein gewöhnliches Papierfilter zu passieren vermochte. Aus solchen Versuchen geht naturgemäh niemals hervor, ob die Auflösung auf aer Bildung eines Ammoniumsilikates beruht. Diese Möglichkeit ist z ar vorhanden, ebensogut konnte aber jediglich eine Peptisation zum Hydrosol eingetreten sein Und in der Tat lehrt denn auch schon die Betrachtung solcher Lösungen mit dem blofen Auge, dab bel dem Prozen in erheblicher Menge kolloide Kieselsäure gebildet wird.

Durch Verfolgung des Lösungsvorganges an Hand von Leitfähigkeitsmessungen 4 ; zeigt sich nun aber, daf das Leitvermögen des Am. moniaks eine beträchtliche Zurahme erfährt,

1) Ber. d. Deutsch. chem. (Ies. 49, 2358 '(1416). $52,601(919) ; 58,1,1509(1920)$.

9) Karsten, Pogg. Ann. 6, 357 (1826).

3) Vgl. Gmetin-Kraut, 7 . Aufl. III, 1, 5. 177.

4) Ueber die ersten Versuche in dieser Richtung

vgi. E. H. Kanter, Diss. (Erianger 1902), siche auch

E. Jordis, Zeitschr. f. Elektrochem 8, 683 . mithin bis zu einen gewissen Grade ionendisperse Kieselsäure gebildet. wird, und zwar in solcher Menge, daB ihre Wirkung in bezug auf das Leitvermögen den hemmcndet Einllut. der ebeufills entstehenden kolloiden Teilchen erheblick überwiegt.

Als Ausgangsmaterial dienten für meine Versuche Kieselsäure-Gele und -Sole, die durch Zerse'zung von Natriumsilikat, Silizumfluorid. Siliziumchlorid und Silizium wasserstoff gewonnen wurden. Auch die bei der Unsetzung yon Dioxo-disiloxan mit Ammoniak entstehende Kieselsäure wurde in den Bereich der Untersuchungen cithezogen. Für die Reinigung diente das Diatysierverfahren.

Es wurde hierfür ein mit Kollodiummembran versehener Dialysator verwendet. Im inneren Gefäb kreiste zur Beschleunigung des Prozesses cin Rührer. Es lieben sich so innerhalb von acht bis zehn Tagen bei den aus Siliziumiteischlorid hergestellten Gelen auch die letzten analytisch nachweisbaren. Spuren von Chlor enifornen. Die Prüfung erfolgte in der Weise, daR das Gel in Natronlauge aus Natrium gelost wurde, die iösung mit chlorfreier Salpetersäurc angesäutert und mit Silbennitrat versetzt wurde. Es wurde das für rein beindene Gel dann noch weiter einige Tage der Dialyse überlassen. Als ein Beweis dafür. dab hier nun wirklich elektrolytfreie Kieselsäure vorliegt. kann die Tatsache angesehen werden, dah das molekulare Leitvermögen der mit solcherart gereinigten Gelen hergestellten Ammoniumsilikatlösungen völlig mit dernjengen von Lösungen übereinstimmi, die durch direkte Umsertung des Ammoniaks mit Silizimmwassersteff gewomnen wurden und bei denen die Anwesenhelt eines Elektrolyten ausgeschlossen ist.

Von ebenso großer Bedeurung wie die Reinheit der Kieselsaure war für die Erzielung einwandfreier Ergebnisse die Indifferenz dos Gefabmaterials. Für alle Unsetzurgen unc Messungen in aninoniakalischer Lösung wurden Quarzgiangefäße benützt. Das Alkali des gewöhnlichen Glases ruft, wie später noch gexeigt wird, ganz erhebliche Störungen hervor. Quarz. glas wird, wie analytische Untersuchung und Messung des Leitvermögens ergab, von $\mathrm{Am}$ moniak der hier benützten Konzentration $[3 n\}$ nicht angegriffen. 
Was das aubere Gepräge der Reaktion Ammoniumhydroxyd-Kieselsaure anlangt, so stellt sie eine langsam verlaufende Zeitreaktion dar. Ihre Geschwindigkeit hängt im wesentlichen vom Wassergehalt und der Zerteilungsform des Kieselsäurehydrates ab. Sie ist am gröBten bei Solen, wo sie in etwa zwölf Stunden beendet ist; bei Gelen schwankt sie je nach dem Wassergehalt von $12-200$ Stunden, sie beträgt bei einem Hydrat mit 13 Proz. Wasser etwa 75 Stunden. Wesentlich geschwinder, nämlich innerhalb weniger Minuten verläuft die Reaktion" solcher Siliziumverbindungen, die sich -.. wie z. B. das Dioxo-disiloxan ${ }^{5}$ ) - unter Wasserstoffentwicklung in Ammoniak zu Ammoniumsilikat umsetzen.

Es gelang nun infolge der Langsamkeit des Prozesses schrittweise den jeweiligen Zustand der Lösung zu verfolgen und festzustellen, wie weit in bestimmten Zeitabständen die Kieselsäure zum Sol peptisiert und wie weit als Salz zum molekulardispérsen Zustand gelöst war.

Für die Bestimmung des Verhältnisses der kolloidisierten zur wahrhaft gelösten Kieselsäuie diente die Tatsache, daß die kolloiden Teilchen gewöhnliche Papierfilter durchlaufen, durch die Zsigmond y'schen Uitrafilter höchster. Feinheit aber vollkommen zurückgehalten werden. Das Verfahren war demnach folgendes:

Die Umsetzung des Kieselsäurehydrates mit. Ammoniak erfolgte in einem gleichzeitig zur Messung der Leitfähigkeit dienenden Quarzglasgefäh. Die Zunahme des Leitvermögens wurde bis zu einer bestimmten Zeit messend verfolgt; unmittelbar nach der letzten Messung wurde die Lösung zunächst durch ein Papierfilter, dann weiter durch ein Membranfilter höchster Feinheit (Nr. 1200 von E. de $\mathrm{H}$ aen f filtriert. Auf diese Weise ließ - sich die wahrhaft gelöste, die kolloìd gelöste Menge und der unlösliche

5) Siliko-Ameisensäureanhydrid. Vgl. Nomenklatur der Siliziumverbindungen bei A. Stock, Ber. d. Deutsch. chem. Ges. 49, 108 (1916).
Rückstand in jedem beliebigen Augenblicke der langsam verlaufenden Reaktion bestimmen: In einem Falle wurde beispielswelse der nach 8 , $24,48,95$ Stunden Versuchsdauer jeweils erreichte Zustand auf diese Art ermittelt und es zeigte sich, daB die in Ultrafiltrat befindliche Kieselsäuremenge bis zur Erreichung eines Maximums ständig größer . wird, die in kolloider Lösung vorhanden gewesene Kieselsäuremenge dagegen bis zur Erreichung eines Minimums ständig kleiner wird. (Vgl. Tabelle l.) Da somit die wahrhaft gelöste Menge auf Kosten der kolloiden Kieselsäure wächst, kann man folgern, dall die Bildung der letzteren, die Peptisation der primäre Vorgang ist, dem als sekundärer die Salzbildung bzw. die Entstehung molekulardisperser Kieselsäure sich anschlieft.

Was die Zunahme des Leitvermögens des hier stets benützten $3 \mathrm{n}$-Ammoniaks bei dem Auflösungsproze $B$ anlangt, so beträgt sie - abgesehen von" später $z u$ besprechenden Ausnahmen - bei $18^{0}$ für $0,1 \mathrm{~g}$ wahrhaft gelöste, d. h. im Uitrafiltrat ermittelte $\mathrm{SiO}_{2} 0,00075$ rezipr. Ohm. Mit anderen Worten ist also der Quotient $\Delta \mathrm{k} / \mathrm{m}=75.10^{-4}$. Dieser Quotient, Q genannt, wird in gleicher Gröbe erhalten, ob das zur Umsetaung benützte Kieselsäurehydrat durch Hydrolyse von Siliziumflisorid, -chlorid, Natriumsilikat oder auch Siliziumwasserstoff entstanden ist. Er bleibt auch dann, wenn man die einmal entstandenen Lösungen sich monatelang selbst überläßit, stets konstant. Irgendwelche nachträgliche Veränderungen scheinen sich somit in den Salzlösungen, die ja stets noch einen Ueberschub an Ammoniak enthalten, 'nicht abzuspielen. Anders dagegen liegen die. Verhältnisse, wenn vor der Umsetzung mit Ammoniak das Kieselsäurehydrat irgendwelchen Einfliussen unterworfen ist.

Hierher gehören insbesondere der freiwillige oder durch Erhitzen hervorgerufene Wasserverlust und die Alterung des Kieselsäuregels.

Tabelle 1 .

Kieselsä uregel mit 12,7 Proz. Wassergehalt. $0,1500 \mathrm{~g}$ in $80 \mathrm{~cm} 3 \mathrm{n}-\mathrm{NH}_{3}, \mathrm{~T}=18^{\circ}$.

\begin{tabular}{|c|c|c|c|c|c|c|c|}
\hline \multirow{2}{*}{ Nr. } & \multirow{2}{*}{$\begin{array}{l}\text { Versuchs- } \\
\text { dauer in } \\
\text { Stunden }\end{array}$} & \multirow{2}{*}{$\begin{array}{c}\text { Gelöste } \mathrm{SiO}_{2} \\
\text { im } \\
\text { Uttrafittrat }\end{array}$} & \multirow{2}{*}{$\begin{array}{c}\text { Ungelöst } \\
\text { g }\end{array}$} & \multirow{2}{*}{$\begin{array}{c}\text { Koltoid gelost } \\
\mathrm{g}\end{array}$} & \multicolumn{3}{|c|}{ In Prozenten } \\
\hline & & & & & gelöst & ungelöst & Kolloid gelöst \\
\hline $\begin{array}{l}1 \\
2 \\
3 \\
4\end{array}$ & $\begin{array}{r}8 \\
24 \\
48 \\
95\end{array}$ & $\begin{array}{l}0,0456 \\
0,0688 \\
0,0842 \\
0,0952\end{array}$ & $\begin{array}{l}0,0195 \\
0,0611 \\
0,0484 \\
0,0417\end{array}$ & $\begin{array}{l}0,0239 \\
0,0209 \\
0,0164 \\
0,0121\end{array}$ & $\begin{array}{l}30,6 \\
46,2 \\
56,5 \\
63,9\end{array}$ & $\begin{array}{l}53,4 \\
41,0 \\
32,5 \\
28,0\end{array}$ & $\begin{array}{r}16,0 \\
12,8 \\
11,0 \\
8,1\end{array}$ \\
\hline
\end{tabular}


Was zuriachst die durch Erhitzen erzielte Wasserabgabe anbetrifft, so hat sie eine Verkleinerung des Quotienten $Q$ zur Folge. Jedoch sinkt dieser Wert nicht kontinuterlich mit gleichmäBig abnehmendem Wassergehalt, son * dern bleibt bis zu einem bestimmten Punkt konstant, um dann sprunghaft $z u$ einer neuen Stufe abzusinken. Von den zahlreichen, in dieser Hinsicht angesteliten Versuchen sei hier eine Reihe wiedergegeben, die mit einem Gel aus Siliziumtetrachlorid ermittelt wurde. (Tabelle II.)

Tabelle II.

Gel aus Siliziumchlorid.

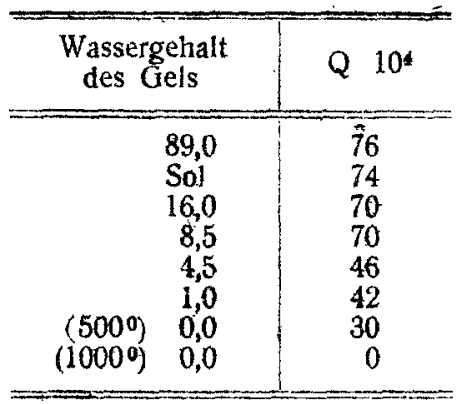

Die Zahlen zeigen, daß die wasserreichen Gele - ebenso auch die Sole - von höchstem Wassergehalt bis herab zu einem Wassergehalt von etwa 15 Proz. den Q-Wert $70-75$ ergeben, daß bei weiterer Verminderung des Wassergehaltes bis zu etwa 2 Proz. der $Q-$ Wert sich um 50 bewegt, um schlieblich bei. stark entwässerten Gelen mit $0,5-2$ Proz. Wasser auf Werte unter 30 und 25 berabzusinken. Wird die Entwässerung durch ștarkes Glühen endlich vollkommen, so erlischt schlieblich die Löslichkeit und đamit auch die Erhöhung der Leitfähigkeit. Es ist zu bemerken, daB einmal bis $z u$ einem bestimmten Grade entwăsserte Gele, deren Wassergehalt nachträglich durch Quellung wieder stark erhöht wurde, auch dann stets den ihrem ursprünglichen Wassergehalt entsprechenden niederen Wert für $Q$ beibehalten und nicht den dem neuerworbenen, hohen Wassergehalt entsprechenden Wert hefern. Nachträgliche Wasseraufnahme vermag also die Veränderungen, die die Kieselsäure durch Wasserverlust einmal erlitten hat, nicht wieder rückgängig zu machen.

AuBer dem Wassergebalt hat auch das Alter des Kieselsäuregels einen Einfluß auf die $\mathrm{Gr} 3 B \in$ des Wertes von Q. Mit Hilfe der Umsetzung in wässerigem Ammoniak und der Ermittelung der Leitfähigkeit der entstehenden Ammoniumsilikatlösungen gelang. es, die Er- scheinung des Alterns des Kieselsäuregels zu verfolgen. Es wurde ein wasserreiches Gel, das durch Hydrolyse von Siliziumtetrachlorid entstanden war, und eine gleichmäBig durch. sichtige: fast wasserklare Gallerte darstellte, der Alterung ohne künstliche Wasserentziehung überlasseǹ. Als Nullpunkt galt die Beendigung des Dialysierprozesses, wo das. Gel durch Abfiltrieren vom umgebenden Wasser befreit wurde. Sein Wassergehalt betrug in diesem Augenblick 95 Proz. Es wurde unter Luftabschlub in einern Exsikkator ohne Trocknungsmittel aufbewahrt. Der Wert für $Q$ ergab sich am frischen Präparat wie erwartet $z u$ 70. Als das Gel 22 Tage alt geworden war, hatte es ioch 91 Proz. Wasser, also nur 4 Proz yerloren. Dagegen ergab sicn aus der Löslichkeî und Leitfahigkeit in Ammoniak der Wert $Q=48$. Nach weiteren 6 Tagen zeigte das Gel die Erscheinung des. Umschlags, es wurde kreideartig weik. Eine jetzt vorgenommene Bestimmung ergab den $Q-$ Wert $=28$. Der Wassergehalt war in diesem Zeitpunkte noch immer sehr hoch, er betrug 83 Proz.

Da ein Teil des Präparates nicht umgeschlagen war; sondern rein äuBerlich unver ${ }^{-}$ ändert erschien, wurde an ihm wiederum eine Bestimmung des $Q$-Wertes vorgenommen und es ergab. sich, daß in diesem klaren, nicht zum Umsehlag geneigten Gele der Wert gleich dem ursprünglichen, für junge, wasserreiche Gele charakteristischen, namlich gleich 75 geblieben war. Der Wassergehalt war noch immer recht beträchtlich, er betrug 75 Proz. Als țach einer sehr langen Zeit, nach 57 Tagen, auch dieser Teil des Gels den Umschiag erfuhr, wurde eine abermalige Bestimmung gemacht. Nun ergab sich auch hier der niedrige, an dem ersten Teile nach dem Umschlag ermitielte Wert $Q=30$.

Das stufenweise Absinken der Leitfähigkeit sowohl beider Wasserentziehung durch Erhitzen wie bei der Alterung ohne wesentlichen Wasserverlust läbt sich dadurch erklären, dab das $\mathrm{Si} \mathrm{O}_{2} \rightarrow$ Molekull Veränderungen erleidet; die als Polymerisation oder Kondensation anzusprecheu sind-und die auch bei der nachträglichen chemischen Unrsetzung, in diesem Falle also der Salzbildung mit Ammoniumhydroxyd erhalten bleiben, indem sich hierbei jeweils entspechend der erreichten Polymerisationsstufe des Kieselsäureanhydrids die zugehörigen Polysilikate bilden.

Hierbei ist besonders beachtenswert, dak der im Laufe der Alterung auftretende Umschlag das äuBere Kenuzeichen für die Erreichung einer weiteren Polymerisationsstufe ist, wobei es natür- 
lich zunächst unbewiesen bleibt, ob die molekularen Veränderungen eine direkte Ursache für das Zustandekommen des Umschlags bilden. Insofern scheint dieses nicht der Fail zu sein. als nămlich der Umschlag dann nicht von einem Absinken des Q-Wertes begleitet ist. wenn er in sehr kurzer Zeit. etwa in zwei Tagen, dadurch herbeigeführt wird. daB man dem Gel über konzentrierter Schwefeisäure das Wasser entzieht. Es bleibt in solchen Fallen $Q=75$, d. h. eine Alterung hat noch nicht stattgefunden, obwohi bereirs Wasserabgabe erfolgte und der Umschlag eingetreten war.

Wir werden also ari der z. Zt. gültigen Etklarung des Unschlags festhalten: Dei rein äulerliche Vorgung beruht auf der Bildung winziger Hohlrăune, die mit Wasserdampf bzw. Luft getüllt sind. Dieses Gemisch hat einen anderen Brechungsexponeuten als das umgebende Gemisch Kieselsäure-fiüssiges Wasser.

Möglich wäre auch, daf das Eintreten des Unschlags und die Erreichung der Polymerisationsstufe mit dem $Q$-Wert 25 nur ein zufäliges Zusammentreffen wäre. Sebr wahrscineinlich scheint mir das nach unseren Versucben allerdings nicht, sofern man eben nur deri ,fremwillig" eintretenden Umschlag in $\mathrm{Be}^{-}$ tracht zieht, der bei noch sehr hohem Wassergehalt erst in vorgerïcktem Alter des Geles eintritt. Es mag daran erinnert werden, dab van Bemmelen den Umschlag durch eine Umwälzung im Bau des Gels, eine neue Koagulation oder Gelbildung $z u$ erkiären versuchte. Gegen diese Auffassung sprach sich Bütschlí aus, indem er hervorhob, dab diese Koagulation durch. Wasserzusatz beliebig wieder rückgängig gemacht und beim Eintrocknen beliebig wieder hervorgerufen werden könne. Aus unserem Befunde folgt nun, daB bei dem langsam aiternden Gele der Umschlag doch von einer „Umwälzung" in Bau, allerdings in anderer Hinsicht als van Bemmelen annahm, begleitet sein kann, und zwar einer solchen, die. wie oben hervorgehoben wurde, durch nachträgliche Wasseraufnahme nicht wieder rïckgängig gemacht $z u$ werden vermag. Denn wie schon betont, andert sich der einmal erreichte Q-Wert nicht wieder, wenn auch der Wassergehalt später künstlich wieder vergrbbert wird.

Die Größe des Quotienten $Q$ hängt auBer yom Wassergehalt und den eben besprochenen Erscheinungen des Alterns auch noch von der

6) O. Butschli, Untersuchungen tiber die Mikrostruktur der Kieselsauregallerten (Heidelberg 1909), 370.
Entstehungsbedingung des Kieselsäurctels ab. Wit fanden, dak bei der Hydrolyse des Silizum. fluorides mit Wasser von $100^{\circ}$ ein Gel gebildet wird, das in vieien Eigenschaften sich erheblich von dem normalen Gel, wie es aus dem fluorid oder Chlorid be $0^{\circ}$ oder Zinmertemperarur entsteht, unterscheidet. Dieses Gel liefert in sehr wasserreichem und frischem Zustande den $Q-$ Wert $=25$, gealtert oder künstlich entwässert den Wert 13. Hier liegt also vermutlich von vornherein eine höher polymerisierte Form der Kieselsäure vor, die unter denselben Einflüssen, wie sie oben für die gewöhnliche Kieselsäure gekennzeichnet wurden, auch dieselbe Art der Veränderung erleidet.

In chemischer Beziehung unterscheidet sich diese ,b-Kieselsäure" von der gewöhnlichen , a-Säure" durch ihr geringeres Reaktionsvermögen gegenüber Flubsäure und Alkalilange. Beispielsweise wird von fünfprozentiger $\mathrm{NaOH}$ bei $18^{\circ}$ die a-Säure in 2 Stunden bis auf 5 Proz. gelost. wogegen die b-Säure fast unloslich ist, von ihr verbleibt ein Rückstand von 83 Proz.

Sehr viel geringe ist auch das Adsorptionsvermögen der $b$ - Säure gegeniuber Methylenblati. Die gewöhnliche Kieselsäure wirỏ, wie bekanat; durch den Parbstoff schön dutikelblau angefarbt, die b-Säure dargegen-betommt nur einen gans schwachen hellblatien Schimmer.

Das Vethalten der b-Säure erinnert in gewisser Hinsicht an die $b$-Zinnsäure, dis der a-Zinnsäure gegenüber ähnliche Unterschiede aufweist, Es sei daran erinuert, dab Mecklenbursis durch Eingielen von Stannisulfat in Wasser von $100^{\prime \prime}$ eine typische b-Zinnsäure erhielt, die sich ebenso wie die unter atmlichen Bedingungen erhattene b-Kieselsäure durch ihr geringeres Absotptionsvermogen und thre kleinete Rediktionsfähigkeit gegenüber Salzsäure von der a-Sảure unterscheidet. Mecklenburg erklärt das verschedene Vernalten der beiden Formen durch die verschiedene Größe der Primärteilchen. Im Sinne dieser Theorie werden wir ohne Bedenken auch für die $b$-Kieselsäure gröBere Primärteilchen annehmen können, um den Unterschied im Verhalten gegenüber der gew öhnlichen Kieselsäure zu erkiaren.

Was die mittelst der geschiluerten Reaktionen entstehenden Amronium-Silikatiösungen selbst anbetrifft, so sind sie nach der Ultra filtration vollsommen 'klar. Sie zeigen das

T) W. MEthlentyurg, Zeitschr f, anorg, Chem. 74, $207(1912)$. 
Tyndallphänomen richt und sind unbegrenzte Zeit balthar. Thre Leitfăhigkeit verändert sich nicht, es tritt also keine nachträgliche Bildung kolloider Kieselsäure dur ch Hydrolyse ein. Diese Tatsache deuret darauf hin, dab die entstandene
Verbindung kein einfaches Silikat, sondern eine komplex Verbindung darstellt.

Ueber Untersuchungen, die sich mit dieser Frage befassen, wird demnächst gesondert berichtet werden.

\title{
Ueber das Gleichgewicht Gelatine-Saizsäure.
}

\author{
Von Robert Wintgen (Göttingen) und Karl Krüger (Bonn).
}

(Eingegangen am 27, Oktober 1930.)

Die Fähigkeit der Gelatine und der Eiweifkörper im allgemeinen, Saizsäure zu binden, ist bereits häufig untersucht worden. Von neueren Arbeiten, die sich mit diesem Gegenstand beschäftigen, seien die Verôffentlichungen Paul i's und seimer Schüler (s.u.), die von Procter (s. u.) and Jacques Loebi) genannt. Wo.Paulia hat tie Etgebnisse seiner diesbezuglichen umfangreichen und bedeutsamen Untersuchungen in der kürzlich erschienenen ersten Hälfte seiner Kolloidchemie der Eiweißhöryer zusammengefabt. Eine Zusammenstellung der älteren Literatur finder sich bei R. Haber ${ }^{3}$ ) und T. B. Robert . son\%. Da zur Untersuchung der Bindung von Elektrolyten an Eiweißkörper die gewöhnlichen analytischen Hilfsmittel wegen Storung des Gleichgewichts in der Regel nicht anwendbar sind, werden neuerdings hauptsächlich physikalisch-chemische Methoden zur Bestimmung der ungebundenen Salzsăure bzw. der Konzentration der freien Wasserstoffionen angewandt. Von diesen Methoden verdient die heute allgemein übliche Methode der Gasketten zweifellos vor alien andern den Vorzug. Wir haben bei schon einige Jahre zurückliegenden, anfänglich mit einem andern Ziel unternommenen Untersuchungen, über die der eine $\left.{ }^{5}\right)$ von uns seinerzeit. kurz berichtete, uns der Methylazetatkatalyse bedient. (F. A. Hoffmann $n^{6}$ und $O$. Cohnheim maben die

1) Jacques l.oeb, Joum. of Gen. Physlol. 1, 237, 363 (1919).

2) Wo. Pa 1 i, Kolloidchemie der Eiweißkorper, 1. Hälte (Dresden u. Leipzig 1920).

3) R. Hober, Physikalische Chemie der Zelle und der Gewebe, 4. Aufl. (Leipzig 1. Berlin 1914)

*) T. B. Robertson, Die physikalische Chemie der Proteine (Dresden u. Leipzig 1912).

5) R. Wintgen, Ueber das Gleichgewicht GeIatine-Salzsäure. (SB, d. Niederrhein. Ges. fïr Natur und Heilkunde in Bonn, Chemische Abteflung 1915; Collegium 1916, 201.)

6) F A. Hoffmann, Zentralbl. 1 klin. Medizin 1889,773, u. 1890,521 .

7) O.Cohnheim, Zeirschr. f. Biol 39,489 (1896).
Wasserstoffionenkonzentration in Saizsäure-Gelatinegemischen mit Hilfe der Katalyse der Robrzuckerinversion.)

Die Gelatine (Marke Non plus ultra) stammte aus den Deutschen Gelatinewerken A. - G. in Hochstam Main. Zur Bestimmung des Wassergelialtes der luftrocknen Gelatine wurde sie bei $110^{\circ}$ bis zur Gewichtskonstanz getrocknet. Wasserverlust in Pron. 17,27 und 17,23, im Mittel 17,25. Die folgenden Angaben beziehen sich der Einfachheit halber, wenn nicht ausdrïcklich anders bemerkt, stets auf lufttrockne Gelatine. Der Aschengehait betruy 1,66 Proz. Die Gelatine seagierte schwach sauer, $1 \mathrm{~g}$ in $\mathrm{CO}_{2}$. freiem Wasser gelöst verbrauchte mit Phenolphthalein als Indikator (Umschlag unscharf) 3,$34 ; 3,66 ; 3,32 ; 3,69$, im Mittel $3,51 \mathrm{~cm}$ $0,05 n \mathrm{NaOH}$. Diese Azidität, die natürlich bei den Titrationen der spăteren Messungen mit zum Ausdrick kommt, ruft selbst keine Beschleunigung der Esterverseifung hervor, wie aus folgenden Vergleichsversuchen ersichtlich. 2 con einer fünfprozentigen bei $25,0^{\circ}$ im Thermostaten stehenden Methylazeiallösung verbriuchten nach

$\begin{array}{ccccc}0 & 23 & 286 & 630 & 1540 \text { Stunden } \\ 0,00 & 0,01 & 0,82 & 3,14 & 13,51 \quad \mathrm{~cm}\end{array}$

$0,05 \mathrm{n} \mathrm{Na} \mathrm{OH} .2 \mathrm{ccm}$ derselben Lósung, aber unter Zusatz von 2 Proz. Gelatine verbrauchten untèr denselben Bedingungen nach

$\begin{array}{ccccc}0 & 23 & 286 & 630 & 1540 \text { Stunden } \\ 0,15 & 0,15 & 0,19 & 0,28 & 0,41 \mathrm{ccm}\end{array}$

0,05 n NaOH. - Der Gelatinezusatz scheint also im Gegenteil bemerkenswerter Weise eine beträchtliche Verlangsamung der Reaktions. geschwindigkeit hervorzurufen.

Die Versuchsanorduung für unsere Messungen war folgende ${ }^{3}$ ):

In einem $100 \mathrm{ccm}$ fassenden Meßkölhchen wurden $0 ; 0,1 ; 0,2 ; 0,5 ; 1 ; 2 ; 3 ; 4 ; 5$ und

8) Ostwald - Luther, Hand- und Hilfsbuch zur Ausführung physiko-chemischer Messungen, 3. Aufl. (Leipzip 1900), 524. 